\title{
The Vascularised Groin Chamber: A Novel Model for Growing Primary Human Liposarcoma in Nude Mice
}

\author{
Daniel Johannes Tilkorn ${ }^{\mathrm{a}, \mathrm{c}}$, Sammy Al-Benna ${ }^{\mathrm{a}}$, Joerg Hauser ${ }^{\mathrm{a}}$, Andrej Ring ${ }^{\mathrm{a}}$, \\ Lars Steinstraesser ${ }^{\mathrm{a}}$, Adrien Daigeler ${ }^{\mathrm{a}}$, Inge Schmitz ${ }^{\mathrm{a}}$, \\ Hans Ulrich Steinau ${ }^{\mathrm{a}}$, Ingo Stricker ${ }^{\mathrm{b}}$
}

\begin{abstract}
Background: The preclinical development of anti-sarcoma drugs has been primarily based on the subcutaneous transplantation of xenografts. Transplant survival remains an obstacle of current models which has been attributed to the period of hypoxia after transplantation. We hypothesized that primary soft tissue sarcoma models with an intrinsic tissue engineered vascular supply would be easily reproducible. The aim of this study was to establish a model of primary human soft tissue sarcoma with an intrinsic vascular supply.
\end{abstract}

Methods: Primary soft tissue sarcoma cells from resected human liposarcomas isolated and divided into tumour fragments were transplanted into a silicon chamber, placed around the superficial epigastric vessels in mice. Sarcoma xenograft samples were analysed histomorphologically (light/electron microscopy and immunohistochemistry).

Results: All primary soft tissue sarcoma transplants engrafted,

Manuscript accepted for publication April 5, 2012

\footnotetext{
${ }^{a}$ Operative Reference Centre for Soft Tissue Sarcoma, Department of Plastic Surgery, BG University Hospital Bergmannsheil, Ruhr University Bochum, Buerkle-de-la-Camp-Platz 1, 44789 Bochum, North Rhine-Westphalia, Germany

bInstitute of Pathology, BG University Hospital Bergmannsheil, Ruhr University Bochum, Buerkle-de-la-Camp-Platz 1, 44789 Bochum, North Rhine-Westphalia, Germany

${ }^{\mathrm{c} C}$ Corresponding author: Daniel Tilkorn, Department of Plastic Surgery, BG University Hospital Bergmannsheil, Ruhr University Bochum, Buerkle-de-la-Camp-Platz 1, 44789 Bochum, North Rhine-Westphalia, Germany. Email: d.tilkorn@web.de
}

doi:10.4021/wjon496w leading to solid tumours within 3 weeks. Histological and immunohistochemical staining confirmed the mouse xenografts as identical high grade liposarcomas compared to original tumour tissue.

Conclusions: This study established a reproducible xenograft model of primary human liposarcoma. This animal model could be of high value for studying human soft tissue sarcomas and their therapy.

Keywords: Angiogenesis; Soft tissue sarcoma; Tissue engineering; Xenograft

\section{Introduction}

Liposarcomas are tumors derived from primitive mesenchymal cells that undergo adipose differentiation and constitute approximately $10 \%$ of all soft tissue sarcomas. Liposarcomas are predominantly a disease of adulthood with peak incidence around the 5th to 6th decade of life and with a slight predominance toward males. The extremities are the most common primary site, accounting for about $40 \%$ of cases. Nearly one-third of cases arise in visceral spaces, and there is an association between site of primary tumor and histologic subtypes [1]. Histologic grade is one of the most important predictors of outcome, with low-grade myxoid tumours having significantly better survival rates compared to the round-cell, pleomorphic, and dedifferentiated subtypes [2-5]. Complete surgical resection remains the mainstay of local therapy, but adjuvant radiation therapy is effective at controlling microscopic residual disease after surgical resection [6-10]. Local tumour recurrence and the development of distant metastases mainly into the lungs remain unsolved clinical problems in the treatment of soft tissue sarcomas [5]. Metastatic disease becomes evident within the first $2-3$ years after initial diagnosis and is the main cause of mortality in these patients [11]. The use of chemotherapy for treatment of liposarcoma is controversial, but myxoid tumors appear to respond better than dedifferentiated or well-differentiated tumors $[3,7,12]$. Despite an aggressive multi disciplinary treatment (surgery, chemotherapy and radiation therapy), the 


\section{Mouse Tissue Engineering Chamber}

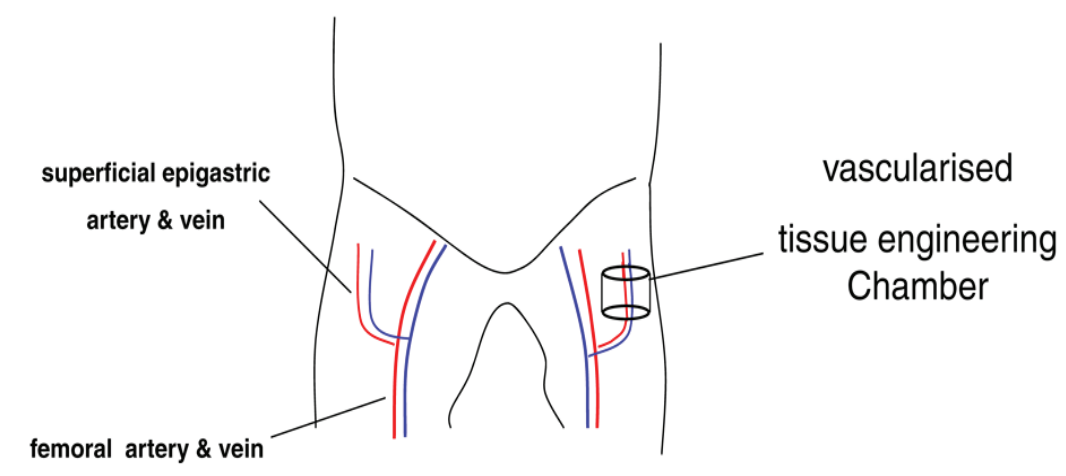

Figure 1. Mouse vascularised tissue engineering groin chamber model for growing primary human liposarcoma.

rate of recurrence of more than $50 \%$ remains very high and results in diffuse metastatic disease and the death of the patients [13]. Treatment for sarcomas has lagged behind more common epithelial cancers, and survival from the high grade liposarcomas has remained unchanged for several decades $[14,15]$. The mechanisms associated with sarcoma development remain largely unclear because of the rarity of the disease, its large number of histological subtypes and its varied clinical behaviour [14, 15]. As such, preclinical models to dissect mechanisms underlying sarcoma development, progression and treatment are greatly needed $[14,15]$.

Subcutaneous and orthotopic xenografts of human tumours in nude mice are an accepted model for in vivo biological and preclinical studies [14-19]. Xenotransplantation has become a widely used tool both to demonstrate the tumourigenicity of cells and to test the efficacy of therapeutic interventions in vivo [20]. The stability and comparability regarding phenotype, differentiation and characteristics of malignancy are important prerequisites of any tumour model. The subcutaneous implantation of tumour fragments into immunocompromised nude mice is a widely accepted model for the study of various tumours $[14,15,17,19,21]$ but there are limited primary sarcoma models due to difficulties in establishing reproducible xenograft models of primary human soft tissue sarcoma $[17,19,21]$. We hypothesized that hypoxic conditions after transplantation may in part be responsible for the cytological instability of the xenotransplants. We hypothesized that primary soft tissue sarcoma models with an intrinsic tissue engineered vascular supply would be easily reproducible. The aim of this study was to establish a reproducible xenograft model of primary human soft tissue sarcoma with an intrinsic tissue engineered vascular supply in nude mice.

\section{Materials and Methods}

\begin{abstract}
Animals
Six weeks old, sexually mature NMR nude mice (Harlan Winkelmann GmbH, Borchen, Germany) weighing about 20 - $25 \mathrm{~g}$ were used in the present study. Animals were housed in ventilated racks with controlled humidity and temperature $\left(20 \pm 2{ }^{\circ} \mathrm{C}\right)$ under pathogen-free conditions and a $12 \mathrm{~h}$ lightdark photoperiodicity and. Food and water as well as boxes, bedding, were sterilised. The surgical procedures used for the inoculation of the cells to give rise to solid tumours and for subsequent removal and transplantation of tumours was conducted under general anaesthesia and steril conditions. All experimental procedures were carried out under the guidelines and with the permission of the ethics committee of the Ruhr University Bochum. Animal care and manipulation adhered to institutional guidelines and the Guide for the Care and Use of Laboratory Animals [22]. Written informed consent of the patient and the permission of the ethics committee of the Ruhr University of Bochum were obtained for the use of tumour specimens.
\end{abstract}

\section{Isolation of sarcoma specimens for xenotransplantation}

On the day of surgery soft tissue sarcoma samples were directly transferred from the operating theatres to the laboratory. Following the tumour ablation tumour samples were taken under sterile conditions at a representative area of the original tumour mass. The sample was then divided into two adjacent parts, one for further histology (conventional light/ electron microscopy and immunohistochemistry) and the other for the in vivo experiment. The tumour tissue was cut 

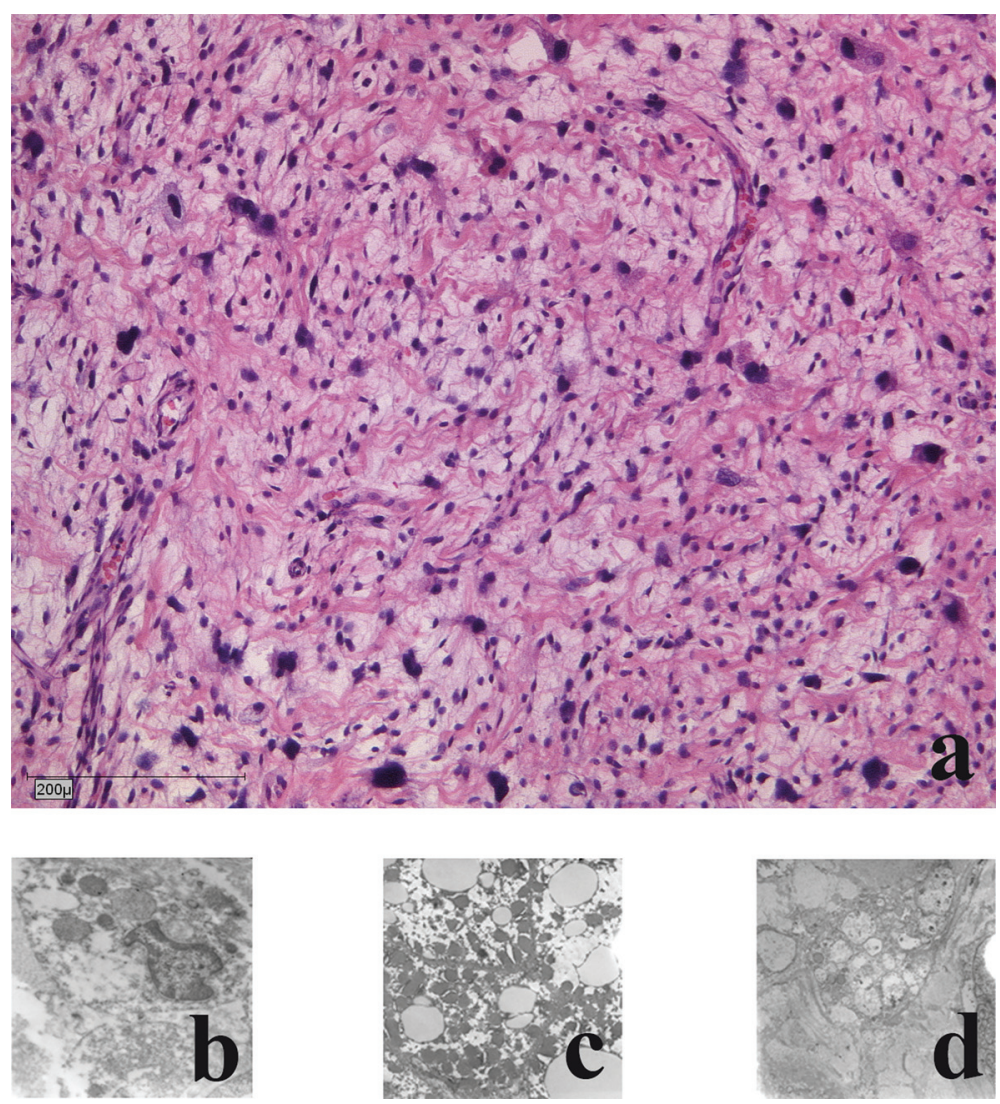

Figure 2. Histological and electron-microscopic morphology of the primary tumour: a) haematoxylin and eosin staining; b) Nuclear morphology demonstrates large atypical cell nuclei with heterochromic prominent nucleoli; c) Cytoplasm: few reticular endoplasmic reticuli with few dark elongated elliptical mitochondria; d) Lipid vacuoles: few with no accumulation of glycogen.

into 1 - $2 \mathrm{~mm}$ fragments prior to transplantation.

\section{Vascularised groin chamber and in vivo sarcoma xeno- graft model}

Tissue engineering chambers $(\mathrm{n}=6)$ consisting of $5 \mathrm{~mm}$ of length of silicone laboratory tubing $(3.35 \mathrm{~mm}$ internal diameter, $4.4 \mathrm{~mL}$ volume; Dow-Corning Corp., Midland, MI) were inserted into the groins of the mouse as previously described [23]. The animal was placed in a supine position, after the exposure of the groins of the mouse a longitudinal incision along the medial thigh was performed. The inguinal fat pad and the superficial epigastric artery and vein were dissected for a distance of $1 \mathrm{~cm}$ from their origin at the femoral vessels (Fig. 1).

The tissue engineering chamber was then placed around the vessels. The chamber was secured to the underlying muscle with a 10-0 nylon microsuture. The longitudinal slid and proximal aperture of the chamber was sealed with bone wax (Ethicon, Somerville, NJ). Care was taken to avoid occlusion of the vascular pedicle. Tumour fragments suspended in Matrigel ${ }^{\circledR}(\mathrm{BD}$ Bioscience, Palo Alto, CA, US) were insert- ed into the chamber. At $37{ }^{\circ} \mathrm{C}$ the matrix solidifies and thus ensures that the cells remain in situ. Then the distal aperture of the chamber was also sealed, creating a semiseald chamber again taking care to not compromise the vascular patency. The construct was carefully returned to the dissected plan within the groin. The wound was closed in layers. The anaesthesia protocol was uneventful in all cases. All animals survived the surgical procedure and recovered well. After recovery animal behaviour returned to normal and remained unsuspicious. No wound complications were noted.

\section{Chamber harvest and specimen assessment}

After an incubation of 3 weeks in vivo in which the animals were allowed to move freely and were fed a standard mouse chew ad libitum the animals were again anaesthetised. The wounds were explored and the chamber (tissue engineering chamber) was exposed. In the chamber the vascular patency was recorded. The vascular pedicle was cut outside the chamber and the chamber retrieved from the implantation site. The outside of the chamber was freed from surrounding fibrous capsular and the chamber reopened. The newly 

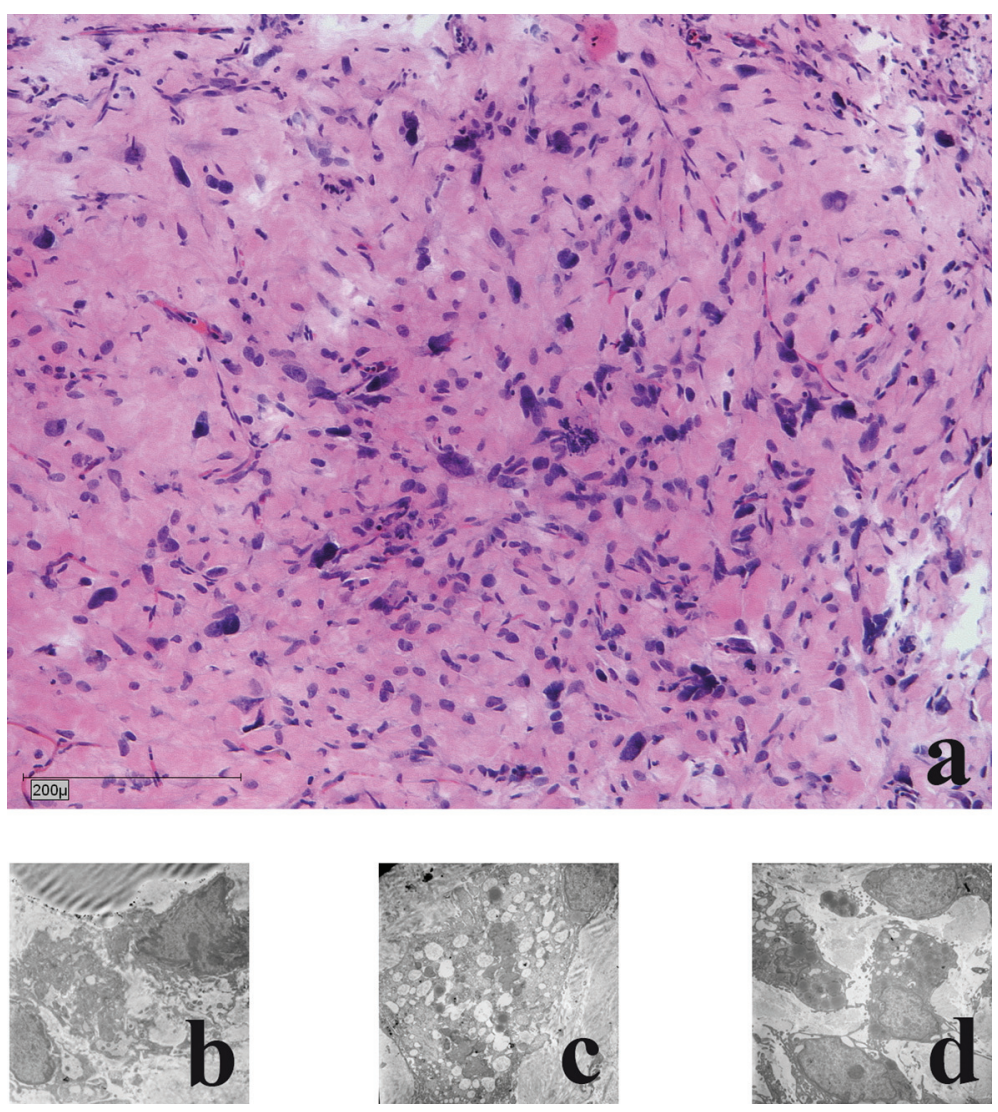

Figure 3. Histological and electronmicroscopic morphology of the xenograft: a) haematoxylin and eosin staining; b) Nuclear morphology demonstrates large oval atypical cell nuclei with heterochromic prominent nucleoli; c) Lipid vacuoles: few light and dark vacuoles with no accumulation of glycogen; d) Cytoplasm: few reticular endoplasmic reticuli with few dark elongated elliptical mitochondria.

formed tissue was then carefully removed. Tissue specimens were photographed and the tissue was divided into two sections one for histological assessment the other one for electron microscopy. Tumour pathology was analyzed by histological and immunohistochemical staining.

\section{Histological assessment}

Tumour samples were placed in 10\% formaldehyde. A minimum of five individual sections of the primary tumour were assessed. The primary tumour and the xenotransplant counterpart were sectioned into $5 \mu \mathrm{m}$ thick sections.

Histological sections were deparaffinised, rehydrated and stained with haematoxylin and eosin following standard procedures.

\section{Electronmicroscopy}

Primary tumour and the xenotransplant specimens were fixed in 2\% glutaraldehyde and embedded in Epon 812. For contrast uranyl acetate and lead citrate were added to the ultra thin sections. Samples were analysed, special focus was placed on differentiation criteria [1]. Microscopic features of the tumours such as cellularity, growth pattern, cytomorphology, vascularity, invasiveness, degree of differentiation, and necrosis were assessed.

\section{Results}

Diagnosis of the primary human liposarcomas was confirmed by independent reference histology. Tumour diagnosis and classification were determined according to WHO guidelines [1].

\section{Histological and electronmicroscopic morphology of the primary tumours}

The liposarcomas showed a pleomorphic growth pattern with atypical mitoses and a marked increase in nuclear and cell changes. Bizarre pleomorphic lipoblasts were seen next to spindle shaped tumour cells, even some giant cells were found. Myxoid changes were rare and tumour necrosis was seen in $15 \%$ of areas. Pancytokeratines (MNF116), EMA, 
smooth muscle actin, desmin, S100 and CD34 were negative (Fig. 2a).

Electron microscopic analysis showed malignant cells with mostly decreased rough endoplasmic reticulum and heterogeneity of cells and nuclei size and shape were present. Nuclear irregularities with multiple nucleoli were frequent. Nucleoli were increased in size and number. The sarcomatous cells had highly irregular nuclear profiles. Mitochondria were electron dark and elongated. Golgi complexes were present. No accumulation of glycogen was seen. Mitotic accumulation of lipid globules was a frequent finding. Few giant cells were present. Focally necrotic areas were seen (Fig. 2b-d).

\section{Histological and electronmicroscopical morphology of the xenotransplants}

In all cases $(n=6)$ the liposarcomas in the chamber model demonstrated a moderate, partial severe inflammation with giant cells was detectable. All cases showed circumjacent to the tumour, slight to moderate proliferations of fibroblasts (Fig 3a). Ultrastructural maintenance was good in the tumour grown in the chamber. Heterogeneity of tumour cells and nuclei was seen with irregularities of nuclei. Focally necrotic areas were seen. Heterogeneity of tumour cells and nuclei was seen with irregularities of nuclei and many small nuclei. Cell necrosis and cell disintegrations were common findings (in more than 50\%) (Fig. 3b-d).

\section{Discussion}

Animal models are indispensable tools for the study of liposarcomas as a paucity of clinical samples makes large-scale analysis of human samples challenging. Furthermore, the diversity of sarcoma subtypes complicates analyses of human sarcomas. Until recently, studies of sarcoma biology were limited to human cell lines and xenografted tumours. An in depth understanding of the tumour biology of these rare tumour entities is warranted to develop and improve sarcoma treatment thus demanding for a well characterized and reproducible in vivo tumour model. Xenotransplantation of tumour cells into immune deficient mice has been well recognised and is a useful experimental model in cancer research $[15,17-19,24]$. The subcutaneous skin fold chamber is an established animal model for malignant soft tissue tumours, but the high failure rate after transplantation as well as the instability regarding phenotype, differentiation and characteristics of the xenograft remains an unsolved problem [25-28]. A $50 \%$ take rate of human tumour xenograft has commonly been reported [25-28]. In a large comparative study of human sarcoma xenografts evaluating intraperitoneal and subcutaneous transplantation sites, Hajdu et al. found an overall take rate of $62 \%$. But only $51 \%$ of all tumour specimens grew subcutaneously. $52 \%$ appeared less $13 \%$ better differentiated and only $35 \%$ resembled the primary tumour when compared to the human tumour resection specimen [18]. The intraperitoneal transplantation resulted in an even higher discordance of differentiation. It is well established that in the initial phase after transplantation cells are exposed to a hypoxic environment and rely on diffusion alone for nutrition [25-28]. Hence a diffusion barrier of more than $200 \mu \mathrm{m}$ will hamper cell survival [29]. It can be hypothesised that this initial vulnerable phase can be accounted for the low graft take after xenotransplantation and may also promote the selection of more resistant hence more dedifferentiated tumour cells. The later is well reflected in the observation that on the one hand the subcutaneous xenograft model is more successful with high grade tumours and on the other hand that dedifferentiation of the tumour when compared to the human primary is frequently reported [17-19]. To overcome this obstacle in the present study, a vascularised chamber for cell transplantation was used.

The vascularised chamber was initially developed for cell transplantation in a tissue engineering setting at the Bernard O'Brien Institute of microsurgery [23]. It has been shown to successfully promote cells survival of thymus [30], cardiac muscle [31], myoblasts and various other tissues [3234]. Centred around a vascular pedicle, the chamber allows for early and rapid neoangiogenesis supporting the nourishment of the transplanted cells and reducing the time period of hypoxia [23].

In the present, all of the xenotransplants into the groin chamber resulted in a survival of the transplanted liposarcomas. As expected from the tissue engineering experiments, an increased angiogenesis was also observed after xenotransplantation of tumour tissue within the groin chamber. All groin chambers displayed a dense functional newly formed vascular network. Tumour cells migrated within the matrix of the groin chamber and seemed to be evenly distributed throughout the chamber. A reduction of connective tissue within the tumour with an increased cell number after xenotransplantation has been described previously.

Immortalised tumour cell lines offer an attractive opportunity for cancer research. Their availability and standardised growth pattern make them suitable for routine testing, but their dedifferentiated nature and acquired additional genetic alterations during the immortalisation process may not reflect all aspects of the primary tumour [25-28]. Differences in phenotype of these cell lines often result from an arrest in differentiation process at specific stages [25-28]. Hence, it is consistently difficult to translate the behaviour of these cells in an experimental setting to a human soft tissue sarcoma in vivo. In addition, primary cell lines are known to acquire genetic alterations after tissue culture expansion. Therefore, in the present study we used tumour fragments transplanted directly after tumour resection to best be able to compare the growth pattern to the original human sarcoma. A potential 
disadvantage to the use of these tumour fragments includes the heterogeneity of the tumour fragment composition and therefore lack of standardisation.

No metastases were observed in any experiment. The short three week observation period may explain this. The tissue engineering chamber produced viable and good tumour growth and seems to be a favourable model to analyze early tumour implantation and tumour stromal interactions. It also offers the chance to better assess the tumour angiogenesis early after transplantation. On the other hand the space limitation with in the silicon chamber might hinder extensive tumour growth.

In conclusion, the vascularised tissue engineering chamber offers a suitable experimental sarcoma model. This model is valuable for sarcoma research because clinical samples are relatively rare and treatment regimens have not changed in decades for many sarcoma subtypes. The further development of models, including the vascularised tissue engineering chamber will continue to increase our understanding of soft tissue sarcoma biology. The susceptibility to hypoxia differs between the sarcoma subgroups and the type of soft tissue sarcoma, the vascularised tissue engineering chamber appears to have benefits in successful outcome of xenograft growth, in creating a more vital tumour that may be a more faithful model and on study of tumour stroma interaction and tumour angiogenesis

\section{Acknowledgement}

This study was supported by the Cancer Assosciation North Rhine Westphalia, Germany (Krebsgesellschaft NordrheinWestfalen e.V.). We gratefully acknowledge the tissue engineering techniques which were developed at the Bernard O'Brien Institute of Medical Research and taught to the first author with the support of Professor Wayne Morrison (University of Melbourne, Australia).

\section{References}

1. Fletcher CDM SM, Rydholm A, Coindre JM, Singer S. . Soft tissue tumours: Epidemiology, clinical features, histopathological typing and grading. Lyon: IARC Press 2002.

2. Mazeron JJ, Suit HD. Lymph nodes as sites of metastases from sarcomas of soft tissue. Cancer. 1987;60(8):18001808.

3. Potter DA, Glenn J, Kinsella T, Glatstein E, Lack EE, Restrepo C, White DE, et al. Patterns of recurrence in patients with high-grade soft-tissue sarcomas. J Clin Oncol. 1985;3(3):353-366.

4. Trojani M, Coquet M, Peres P, Coindre JM, Ragni R, Meuge-Moraw C. [Primary leiomyosarcoma of bone. A case with ultrastructural study. Review of the literature]. Arch Anat Cytol Pathol. 1982;30(4):197-201.

5. Vezeridis MP, Moore R, Karakousis CP. Metastatic patterns in soft-tissue sarcomas. Arch Surg. 1983;118(8):915-918.

6. Al-Benna S, Poggemann K, Steinau HU, Steinstraesser L. Diagnosis and management of primary breast sarcoma. Breast Cancer Res Treat. 2010;122(3):619-626.

7. Clarkson P, Ferguson PC. Primary multidisciplinary management of extremity soft tissue sarcomas. Curr Treat Options Oncol. 2004;5(6):451-462.

8. Clarkson P, Ferguson PC. Management of soft tissue sarcomas of the extremities. Expert Rev Anticancer Ther. 2004;4(2):237-246.

9. Daigeler A, Klein-Hitpass L, Stricker I, Muller O, Kuhnen C, Chromik AM, Steinstraesser L, et al. Malignant fibrous histiocytoma--pleomorphic sarcoma, NOS gene expression, histology, and clinical course. A pilot study. Langenbecks Arch Surg. 2010;395(3):261-275.

10. Daigeler A, Kuhnen C, Moritz R, Stricker I, Goertz O, Tilkorn D, Steinstraesser L, et al. Lymph node metastases in soft tissue sarcomas: a single center analysis of 1,597 patients. Langenbecks Arch Surg. 2009;394(2):321-329.

11. Kasper B, Gil T, D'Hondt V, Gebhart M, Awada A. Novel treatment strategies for soft tissue sarcoma. Crit Rev Oncol Hematol. 2007;62(1):9-15.

12. Issels RD, Schlemmer M. Current trials and new aspects in soft tissue sarcoma of adults. Cancer Chemother Pharmacol. 2002;49 Suppl 1:S4-8.

13. Pisters P. Staging and prognosis. In: Pollock RE e, ed. American Cancer Society Atlas of Clinical Oncology: soft tissue sarcomas. Hamiliton, Ontario:: BC Decker Inc. 2002.

14. Steinstraesser L, Hauk J, Jacobsen F, Stricker I, Steinau HU, Al-Benna S. Establishment of a synovial sarcoma model in athymic nude mice. In Vivo. 2011;25(2):165169.

15. Steinstraesser L, Jacobsen F, Schubert C, Gevers K, Stricker I, Steinau HU, Al-Benna S. Establishment of a primary human sarcoma model in athymic nude mice. Hum Cell. 2010;23(2):50-57.

16. Bastert G EH, Usadel KH, Althoff PH, Schwedes U, Steinau U, Klempa I FH. Xenografts of Benign and Malignant Endocrine Tissues in Thymusaplastic Nude Mice and Rats: Development and Function. In: Bastert, GBA S-MH, Fortmeyer HPJ, eds., eds. Thymusaplastic Nude Mice and Rats in Clinical Oncology. Stuttgart: Gustav Fischer Verlag 1981:383-407.

17. Giovanella BC, Stehlin JS, Jr., Williams LJ, Jr., Lee SS, Shepard RC. Heterotransplantation of human cancers into nude mice: a model system for human cancer chemotherapy. Cancer. 1978;42(5):2269-2281.

18. Hajdu SI, Lemos LB, Kozakewich H, Helson L, Beattie EJ, Jr. Growth pattern and differentiation of human soft 
tissue sarcomas in nude mice. Cancer. 1981;47(1):9098.

19. Hattler BG, Jr., Soehnlen B, Seaver NA, Sato P. Heterotransplantation of human malignant neoplasms to the mouse mutant nude. Surg Forum. 1974;25(0):127-129.

20. Scherer WF, Syverton JT, Gey GO. Studies on the propagation in vitro of poliomyelitis viruses. IV. Viral multiplication in a stable strain of human malignant epithelial cells (strain HeLa) derived from an epidermoid carcinoma of the cervix. J Exp Med. 1953;97(5):695-710.

21. Helson L, Das SK, Hajdu SI. Human neuroblastoma in nude mice. Cancer Res. 1975;35(9):2594-2599.

22. Resources IoLA. In: Commission on Life Sciences NRC, ed. Washington, D.C.;: National Academy Press 1996.

23. Cronin KJ, Messina A, Knight KR, Cooper-White JJ, Stevens GW, Penington AJ, Morrison WA. New murine model of spontaneous autologous tissue engineering, combining an arteriovenous pedicle with matrix materials. Plast Reconstr Surg. 2004;113(1):260-269.

24. Steinstraesser L, Schubert C, Hauk J, Becerikli M, Stricker I, Koeller M, Hatt H, et al. Oncolytic designer host defense peptide suppresses growth of human liposarcoma. Int J Cancer. 2011;128(12):2994-3004.

25. Itescu S, Schuster MD, Kocher AA. New directions in strategies using cell therapy for heart disease. J Mol Med (Berl). 2003;81(5):288-296.

26. Kneser U, Kaufmann PM, Fiegel HC, Pollok JM, Kluth D, Herbst H, Rogiers X. Long-term differentiated function of heterotopically transplanted hepatocytes on three-dimensional polymer matrices. J Biomed Mater Res. 1999;47(4):494-503.
27. Muschler GF, Nakamoto C, Griffith LG. Engineering principles of clinical cell-based tissue engineering. J Bone Joint Surg Am. 2004;86-A(7):1541-1558.

28. Steffens L, Wenger A, Stark GB, Finkenzeller G. In vivo engineering of a human vasculature for bone tissue engineering applications. J Cell Mol Med. 2009;13(9B):3380-3386.

29. Folkman J, Hochberg M. Self-regulation of growth in three dimensions. J Exp Med. 1973;138(4):745-753.

30. Seach N, Mattesich M, Abberton K, Matsuda K, Tilkorn DJ, Rophael J, Boyd RL, et al. Vascularized tissue engineering mouse chamber model supports thymopoiesis of ectopic thymus tissue grafts. Tissue Eng Part C Methods. 2010;16(3):543-551.

31. Morritt AN, Bortolotto SK, Dilley RJ, Han X, Kompa AR, McCombe D, Wright CE, et al. Cardiac tissue engineering in an in vivo vascularized chamber. Circulation. 2007;115(3):353-360.

32. Lepore DA, Thomas GP, Knight KR, Hussey AJ, Callahan T, Wagner J, Morrison WA, et al. Survival and differentiation of pituitary colony-forming cells in vivo. Stem Cells. 2007;25(7):1730-1736.

33. Hemmrich K, Thomas GP, Abberton KM, Thompson EW, Rophael JA, Penington AJ, Morrison WA. Monocyte chemoattractant protein-1 and nitric oxide promote adipogenesis in a model that mimics obesity. Obesity (Silver Spring). 2007;15(12):2951-2957.

34. Thomas GP, Hemmrich K, Abberton KM, McCombe D, Penington AJ, Thompson EW, Morrison WA. Zymosaninduced inflammation stimulates neo-adipogenesis. Int J Obes (Lond). 2008;32(2):239-248. 